\title{
Understanding barriers to medication adherence in the hypertensive population by evaluating responses to a telephone survey
}

This article was published in the following Dove Press journal:

Patient Preference and Adherence

26 April 201 I

Number of times this article has been viewed

\author{
Kavita V Nair' \\ Daniel A Belletti ${ }^{3}$ \\ Joseph J Doyle ${ }^{3}$ \\ Richard R Allen ${ }^{4}$ \\ Robert B McQueen' \\ Joseph J Saseen' \\ Joseph Vande Griend' \\ Jay $\vee$ Patel $^{5}$ \\ Angela McQueen ${ }^{2}$ \\ Saira Jan ${ }^{2}$ \\ 'School of Pharmacy, University \\ of Colorado, Aurora, CO, USA; \\ ${ }^{2}$ Horizon Blue Cross Blue Shield of \\ New Jersey, Newark, NJ, USA \\ ${ }^{3}$ Novartis Pharmaceuticals \\ Corporation, East Hanover, \\ NJ, USA, ${ }^{4}$ Peakstat Statistical \\ Services, Evergreen, CO, USA \\ ${ }^{5}$ Care Management International, \\ Marlborough, MA, USA
}

Background: Although hypertension is a major risk factor for cardiovascular disease, adherence to hypertensive medications is low. Previous research identifying factors influencing adherence has focused primarily on broad, population-based approaches. Identifying specific barriers for an individual is more useful in designing meaningful targeted interventions. Using customized telephonic outreach, we examined specific patient-reported barriers influencing hypertensive patients' nonadherence to medication in order to identify targeted interventions.

Methods: A telephone survey of 8692 nonadherent hypertensive patients was conducted. The patient sample comprised health plan members with at least two prescriptions for antihypertensive medications in 2008. The telephone script was based on the "target" drug associated with greatest nonadherence (medication possession ratio [MPR] $<80 \%$ ) during the four-month period preceding the survey.

Results: The response rate was $28.2 \%$ of the total sample, representing $63.8 \%$ of commercial members and $37.2 \%$ of Medicare members. Mean age was 63.4 years. Mean MPR was $61.0 \%$ for the target drug. Only $58.2 \%$ of Medicare respondents and $60.4 \%$ of commercial respondents reported "missing a dose of medication". The primary reason given was "forgetfulness" (61.8\% Medicare, $60.8 \%$ commercial), followed by "being too busy" (2.7\% Medicare, 18.5\% commercial) and "other reasons" (21.9\% Medicare, $8.1 \%$ commercial) including travel, hospitalization/sickness, disruption of daily events, and inability to get to the pharmacy. Prescription copay was a barrier for less than $5 \%$ of surveyed patients.

Conclusion: Our findings indicate that events interfering with daily routine had a significant impact on adherence. Medication adherence appears to be a patterned behavior established through the creation of a routine and a reminder system for taking the medication. Providers should assess patients' daily schedules and medication-taking competency to develop and promote a medication routine.

Keywords: adherence, hypertension, antihypertensive therapy

\section{Background}

Hypertension is a major risk factor for cardiovascular disease. Although antihypertensive medications have been demonstrated to control hypertension, over half of patients stop taking their medications entirely within a year of diagnosis, and only $31 \%$ take at least $80 \%$ of their prescribed medication. ${ }^{1,2}$ A World Health Organization report ${ }^{3}$ estimates that adherence to antihypertensive medications ranges from $52 \%$ to $74 \%$ when adherence is defined as possession of a medication at least $80 \%$ of the time. ${ }^{4,5}$ This World Health Organization report identified nonadherence to medical treatment as a major public health concern, especially in patients with chronic conditions (eg, hypertension). ${ }^{3}$
Correspondence: Kavita $\vee$ Nair School of Pharmacy, University of Colorado, Mail Stop C238, I2850 E

Montview Blvd, Aurora, CO 80045, USA

Tel +30 37242635

Fax +30 37240479

Email kavita.nair@ucdenver.edu 
Medication adherence is defined as the extent to which a patient follows a given therapeutic medication regimen as agreed on in partnership with a health care professional. ${ }^{3}$ In the context of hypertension, medication adherence has been associated with improved health outcomes. ${ }^{6}$ Mazzaglia et al ${ }^{7}$ demonstrated that, in 18,806 newly diagnosed hypertensive patients, those with high medication adherence (considered to be $80 \%$ of the days covered with drug therapy) had $38 \%$ fewer acute cardiovascular events long term compared with patients with low medication adherence. The literature on medication adherence has led to the development of several classifications of factors that influence nonadherence. Over 250 factors have been identified, including patient abilities, beliefs, involvement, practical difficulties, support systems, and provider relationships. ${ }^{8}$

Kingsnorth and Wilkinson ${ }^{9}$ described medication nonadherence as having two components, ie, a purposeful decision-making process wherein patients intentionally choose to stop taking their medication due to beliefs or perceptions about the medication, and a random departure from taking medication due to factors such as forgetfulness, interruption of daily routines, inability to access medications, or lack of reminders. Therefore, medication nonadherence has been reconceptualized as either intentional or unintentional. ${ }^{10}$ This reconceptualization has allowed researchers to expand their focus beyond patient perceptions of medicationtaking to include patients' actual abilities to carry out medication treatment plans.

Harmon et $\mathrm{al}^{11}$ categorized barriers for nonadherence among hypertensive patients as intentional nonadherence barriers, which include knowledge or perceptions of the disease, cost, complexity of antihypertensive regimens, side effects, and unintentional nonadherence barriers, which include forgetfulness and lack of access to medications. Research examining these barriers has shown that intentional nonadherence is based on a misunderstanding of the condition (ie, the patient "assumed blood pressure was normal"), side effects, asymptomatic nature of the disease, cost of medication, complexity of regimen ("concern about having too many medications"), and switching to a generic medication. ${ }^{12,13}$ (Although switching to less costly generic medications could actually improve adherence, in some cases, switching to a generic drug could lead to a real or perceived increase in side effects or a decrease in efficacy which, in turn, could lead to nonadherence). Unintentional barriers are based on forgetfulness ("forgetting to take the medication when the usual routine changes"), availability of transportation to the pharmacy, and having to obtain medications every month. ${ }^{13}$

The primary goal of this study was to conduct a deeper examination of which patient-identified barriers influence adherence for a large sample of commercially-insured and Medicare-insured hypertensive patients, using telephone surveys. Secondary objectives were to determine the distribution of respondents who were taking the various classes of antihypertensive medications, and to compare patient characteristics between commercially-insured and Medicare-insured patients in order to determine whether there were any differences in adherence patterns between those younger and older than 65 years of age and whether type of insurance influenced adherence.

\section{Methods Design}

A telephone survey of 8692 nonadherent hypertensive patients was conducted to identify whether their nonadherence was intentional, unintentional, or a combination of both. Patients were identified using pharmacy claims data from a large health insurance plan in the mid-Atlantic US that included commercial ( $<65$ years) and Medicare members ( $\geq 65$ years). Criteria for participation included age $\geq 18$ years, at least two consecutive prescription fills for an antihypertensive medication between January 1, 2008 and August 31, 2008, from the drug classes of alphablockers, beta-blockers, calcium channel blockers, diuretics (excluding furosemide), angiotensin receptor blockers, angiotensin-converting enzyme inhibitors, antihypertensive combinations, antiadrenergic antihypertensives, and direct renin inhibitors, and enrollment in the health plan at the time of the survey.

From this group, patients with a medication possession ratio (MPR) of $<80 \%$ for at least one of their antihypertensive medications, as determined from pharmacy claims data, were chosen. This MPR was selected because, first, it is a commonly used benchmark of nonadherence, 4,5 and second, an MPR of $<80 \%$ has been associated with increased hospitalizations. ${ }^{14}$ If patients were taking more than one antihypertensive medication, the drug with the lowest MPR in the four-month period preceding the survey was used as the "target" drug to assess whether they could be included in the study sample. If individuals had an MPR $<80 \%$ for more than one antihypertensive medication, the one with the most recent use as identified by the last fill date was designated as the target medication. 
A pilot test of the telephone survey was conducted in the summer of 2008 using a subset of 500 patients. The telephone survey was implemented between October and December 2008 by Care Management International..$^{15}$ Three attempts were made to contact patients who were nonadherent or their dependents (who were permitted to serve as health proxies), and an incoming call line was established for plan members or dependents who wanted to return calls. Calls were made by trained employees of Care Management International using a pilot-tested and validated script that directed respondents to specific follow-up questions about barriers to adherence based on their individual responses to key domain questions. The pilot test revealed that some survey items and skip patterns of questions needed to be modified. Accordingly, a revised script was utilized on members of the pilot sample $(n=500)$ who did not complete the pilot survey in the first round (ie, they could not be reached or they did not have time to complete the survey). Based on the results of the pilot survey, the survey items were modified to create a final pilot-tested and validated survey. The telephone survey averaged eight minutes. Institutional Review Board (IRB) approval was obtained, and patients' responses to the survey were used as a proxy (per IRB process) for implied and informed consent.

\section{Key survey items}

We examined eight domains, ie, confirmation that the respondent had hypertension and was using the target drug, dosing pattern of the target medication, means of purchase of the medication, self-reported nonadherence, barriers to nonadherence, self-reported aids to overcome nonadherence, use of mail-order pharmacy services, and preferred method of receiving educational materials. Follow-up questions were tailored to an individual's response to the key domain question. Selected survey items used to measure key domains and a sampling of follow-up questions are described in the Appendix.

\section{Other measures}

\section{Demographic measures}

Age, gender, and primary subscriber to the health plan (patient or other) as of 2008 were determined for respondents and nonrespondents, as determined from claims (enrollment) data.

\section{Clinical measures}

The Prescription Drug Hierarchical Condition Category (RxHCC) score was determined for each individual in 2008.
This risk score was developed by Centers for Medicare and Medicaid Services for Medicare Part D beneficiaries using data from the Federal Employee Health Plan run by Blue Cross Blue Shield. ${ }^{16}$ The risk score uses diagnosis codes in a base year to predict an individual's prescription costs for the following year. The RxHCC diagnostic classification system categorizes more than 15,000 International Classification of Diseases, Ninth Revision (ICD-9) diagnosis codes into 197 conditions or RxHCCs, and uses these categories in the model, where cost denotes total prescription expenditures and is the outcome variable. Explanatory variables include 24 mutually exclusive age/gender categories, disabled status, and the RxHCCs, which are a vector of diagnostic categories.

\section{Medication use and adherence}

This was determined from pharmacy claims as the total number of antihypertensive medications (including the target medication) an individual was taking, total number of all medications (including antihypertensive medications) an individual was taking, adherence rate of the target medication (as identified by MPR $\geq 80 \%$ ), adherence rate for other antihypertensive medications excluding the target medication, and nonadherence strata in which respondents were categorized, ie, $0 \%-19 \%, 20 \%-39 \%, 40 \%-59 \%$, and $60 \%-79 \%$.

\section{Comorbidities}

Presence of comorbidities in 2008 was determined on the basis of patients' self-reported diagnoses during the telephone conversation, and comprised diabetes, depression, dyslipidemia, chronic kidney disease, congestive heart failure, coronary heart disease, and ischemic stroke.

\section{Statistical analysis}

Univariate descriptive analysis was used to compare respondents and nonrespondents on demographic characteristics (n [\%]), clinical characteristics (mean \pm standard deviation [SD]), medication use and adherence (mean $\pm \mathrm{SD}$ and $\mathrm{n}[\%]$ ), presence of specific comorbidities (n [\%]), medical utilization (mean $\pm \mathrm{SD}$ ), and expenditures (mean $\pm \mathrm{SD}$ ). A similar analysis was used to compare the commercially-insured and Medicare-insured respondents. Univariate analysis was also used to describe differences between commerciallyinsured and Medicare-insured enrollees' responses regarding "confirming hypertension and use of target drug", "dosing pattern of target drug", "source of medication purchase", "self-reported nonadherence", "use of mail-order pharmacy 
services", and "preferred method of receiving educational materials". Differences in specific barriers to adherence and self-reported aids to overcoming adherence between these two groups were assessed, adjusting for age and gender using odds ratios with associated $95 \%$ confidence intervals; $P$ values were reported $(P<0.5$ denotes statistical significance). All analyses were conducted using SAS version 9.2.

\section{Results}

\section{Response rates}

A total of 8692 members were eligible to participate in the study. Of those, 2383 (27.4\%) members completed the entire telephone survey, and another $68(<1.0 \%)$ responded to part of the telephone survey. Thus, the overall response rate was $28.2 \%(n=2451)$, representing $63.8 \%$ of commercial members and $37.2 \%$ of Medicare members.

\section{Respondents versus nonrespondents}

Respondents $(\mathrm{n}=2451)$ were older $(63.4$ years versus 58.6 years), had a greater percentage of females $(54.3 \%$ versus $47.3 \%$ ), and had higher RxHCC risk scores (1.143 versus 1.096) compared with nonrespondents $(n=6241$, see Table 1). Adherence rates for the target drug were similar for both groups at approximately $60 \%$, as was adherence to other antihypertensive medications (approximately 85\%).

\section{Commercially-insured versus Medicare-insured respondents}

As shown in Table 2, Medicare-insured respondents $(\mathrm{n}=887)$ were older ( 75.5 years versus 56.4 years), had a greater percentage of females (64.3\% versus $48.7 \%$ ), and had higher RxHCC risk scores (1.167 versus 1.129) compared with commercially-insured respondents $(n=1564)$. Medicareinsured respondents also had a slightly higher number of antihypertensive medications (2.9 versus 2.4$)$ and total number of medications (11.2 versus 10.2). Adherence rates for the target drug were slightly higher for commerciallyinsured respondents (61.1\% versus 59.9\%), while adherence to other antihypertensive medications was slightly higher for Medicare-insured respondents (87.4\% versus $84.5 \%$ ) among those taking multiple antihypertensive medications. Medicareinsured respondents had higher rates of all comorbidities examined, notably coronary heart disease $(23.8 \%$ versus $10.5 \%)$, congestive heart failure ( $15.3 \%$ versus $6.1 \%)$, chronic kidney disease $(8.9 \%$ versus $4.1 \%)$, dyslipidemia $(19.5 \%$ versus $13.5 \%)$, and diabetes (19.3\% versus $12.3 \%)$.

\section{Distribution of drug classes and adherence strata for target drugs}

Among the target drugs, beta-blockers represented $32 \%$ of those targeted for commercially-insured respondents and

Table I Comparing characteristics of survey respondents with those of nonrespondents

\begin{tabular}{|c|c|c|c|}
\hline & Respondents & Nonrespondents & $P$ value \\
\hline Sample size, $\mathrm{n}$ & 2451 & 6241 & \\
\hline \multicolumn{4}{|l|}{ Demographics } \\
\hline Age, mean (SD) & $63.4(13.68)$ & $58.6(13.45)$ & $<0.0001$ \\
\hline Females, n (\%) & $1332(54.3 \%)$ & $2950(47.3 \%)$ & $<0.00001$ \\
\hline Primary subscribers, n (\%) & 2061 (84.l\%) & $4985(79.9 \%)$ & $<0.0001$ \\
\hline \multicolumn{4}{|l|}{ Risk score } \\
\hline RxHCC score, mean (SD) & $\mathrm{I} .143(0.3212)$ & $1.096(0.3143)$ & $<0.0001$ \\
\hline \multicolumn{4}{|l|}{ Medication use and adherence } \\
\hline Number of all antihypertensive medications, mean (SD) & $2.6(1.50)$ & $2.3(1.40)$ & $<0.0001$ \\
\hline $\begin{array}{l}\text { Number of all medications including antihypertensive } \\
\text { medications, mean (SD) }\end{array}$ & $10.6(6.38)$ & $9.6(6.31)$ & $<0.0001$ \\
\hline Adherence to target medication, mean (SD) & $60.7(13.94)$ & $60.5(14.1 \mathrm{I})$ & 0.6432 \\
\hline $\begin{array}{l}\text { Adherence to all other antihypertensive medications except } \\
\text { the target medication, mean (SD) }\end{array}$ & $85.6(17.1 I)$ & $84.2(17.53)$ & 0.0026 \\
\hline \multicolumn{4}{|l|}{ Comorbidities } \\
\hline Members with diabetes, n (\%) & $363(14.8 \%)$ & $784(12.6 \%)$ & $<0.0001$ \\
\hline Members with depression, n (\%) & $69(2.8 \%)$ & $197(3.2 \%)$ & 0.4057 \\
\hline Members with dyslipidemia, n (\%) & $384(15.7 \%)$ & $838(13.4 \%)$ & 0.0069 \\
\hline Members with chronic kidney disease, n (\%) & $143(5.8 \%)$ & $254(4.1 \%)$ & 0.0004 \\
\hline Members with congestive heart failure, n (\%) & $232(9.5 \%)$ & $407(6.5 \%)$ & $<0.0001$ \\
\hline Members with coronary heart disease, n (\%) & $376(15.3 \%)$ & $793(12.7 \%)$ & 0.0012 \\
\hline Members with ischemic stroke, n (\%) & $50(2.0 \%)$ & I I8 (I.9\%) & 0.6492 \\
\hline
\end{tabular}

Abbreviations: SD, standard deviation; RxHCC, Prescription Drug Hierarchical Condition Category. 
Table 2 Comparing characteristics of commercially-insured survey respondents with those of Medicare-insured respondents

\begin{tabular}{|c|c|c|c|}
\hline & $\begin{array}{l}\text { Commercially-insured } \\
\text { respondents }\end{array}$ & $\begin{array}{l}\text { Medicare-insured } \\
\text { respondents }\end{array}$ & $P$ value \\
\hline Sample size, $n$ & 1564 & 887 & \\
\hline \multicolumn{4}{|l|}{ Demographics } \\
\hline Age, mean (SD) & $56.4(10.84)$ & $75.7(8.50)$ & $<0.0001$ \\
\hline Females, n (\%) & $762(48.7 \%)$ & $570(64.3 \%)$ & $<0.0001$ \\
\hline Primary subscribers, n (\%) & II 74 (75. I\%) & $887(100 \%)$ & $<0.0001$ \\
\hline \multicolumn{4}{|l|}{ Risk score } \\
\hline RxHCC score, mean (SD) & $1.129(0.3178)$ & $1.167(0.3260)$ & 0.0046 \\
\hline \multicolumn{4}{|l|}{ Medication use and adherence } \\
\hline Number of all antihypertensive medications, mean (SD) & $2.4(1.45)$ & $2.9(1.52)$ & $<0.0001$ \\
\hline $\begin{array}{l}\text { Number of all medications including antihypertensive } \\
\text { medications, mean (SD) }\end{array}$ & $10.2(6.61)$ & $11.2(5.92)$ & 0.0007 \\
\hline Adherence to target medication, mean (SD) & $61.1(13.80)$ & $59.9(14.17)$ & 0.0424 \\
\hline $\begin{array}{l}\text { Adherence to all other antihypertensive medications } \\
\text { except the target medication, mean (SD) }\end{array}$ & $84.5(17.69)$ & $87.4(16.05)$ & 0.0004 \\
\hline \multicolumn{4}{|l|}{ Comorbidities } \\
\hline Members with diabetes, $\mathrm{n}(\%)$ & $192(12.3 \%)$ & I7| (19.3\%) & $<0.0001$ \\
\hline Members with depression, $\mathrm{n}(\%)$ & $4 \mathrm{I}(2.6 \%)$ & $28(3.2 \%)$ & $0.44 \mid 4$ \\
\hline Members with dyslipidemia, n (\%) & $211(13.5 \%)$ & $173(19.5 \%)$ & $<0.000$ I \\
\hline Members with chronic kidney disease, $n(\%)$ & $64(4.1 \%)$ & $79(8.9 \%)$ & $<0.0001$ \\
\hline Members with congestive heart failure, $\mathrm{n}(\%)$ & $96(6.1 \%)$ & $136(15.3 \%)$ & $<0.0001$ \\
\hline Members with coronary heart disease, $\mathrm{n}(\%)$ & $165(10.5 \%)$ & $211(23.8 \%)$ & $<0.000$ I \\
\hline Members with ischemic stroke, $\mathrm{n}(\%)$ & $18(1.2 \%)$ & $32(3.6 \%)$ & $<0.0001$ \\
\hline
\end{tabular}

Abbreviations: RxHCC, Prescription Drug Hierarchical Condition Category; SD, standard deviation.

$24 \%$ of those targeted for Medicare-insured respondents (Figure 1). Diuretics represented the target drug for $21.4 \%$ of commercial and $18.1 \%$ of Medicare respondents, followed by angiotensin-converting enzyme inhibitors (18.2\% commercial and $15.9 \%$ Medicare), and angiotensin receptor blockers ( $13.1 \%$ commercial versus $15.9 \%$ Medicare). None of the respondents were taking calcium channel blockers as the target drug.

A majority of respondents in both groups $(58.6 \%$ Medicare and $60 \%$ commercial) had adherence rates between $60 \%$ and $79 \%$ for their target drug. About a third of respondents in each group had adherence rates between $40 \%$ and $59 \%$ for their target drug.

\section{Key survey domain responses}

\section{Confirming hypertension and use of target drugs}

More than three quarters of both Medicare-insured (78.7\%) and commercially-insured (82.4\%) respondents confirmed that they were using the target drug for hypertension. About $12 \%$ of the Medicare group and $8 \%$ of the commercial group reported not taking the target drug, but were taking an alternative drug to manage their hypertension, while $<5 \%$ in each group reported not taking their target drug anymore. Over $96 \%$ of all respondents claimed that their blood pressure was under control.

\section{Dosing pattern of target drug}

A majority of respondents in both groups $(72.2 \%$ commercial and $80.32 \%$ Medicare) took their target drug once a day. Between $17 \%$ and $24 \%$ of each group reported taking their target medication twice a day, while $<5 \%$ reported taking it three times a day or had some other dosing schedule. Over $66 \%$ of each group reported taking their medication in the morning, $6 \%$ said they took it in the afternoon, and $28 \%$ said they took it in the evening.

\section{Self-reported nonadherence}

There were $58.2 \%$ Medicare-insured and $60.4 \%$ commerciallyinsured respondents who reported missing a dose of their target medication $(P=0.2892)$. For those who reported not missing a dose of their medication, $13.1 \%$ of the Medicareinsured group and $16.3 \%$ of the commercially-insured group did report picking up their prescriptions a few days after running out of pills $(P=0.1822)$.

\section{Barriers to adherence}

The primary self-reported barrier to adherence appeared to be forgetfulness (64.9\% Medicare and $73.32 \%$ commercial, $P=0.0320$, Table 3 ). A closer examination of why respondents forgot to take their medication revealed that $23.3 \%$ of commercially-insured and $12.9 \%$ of Medicare-insured 


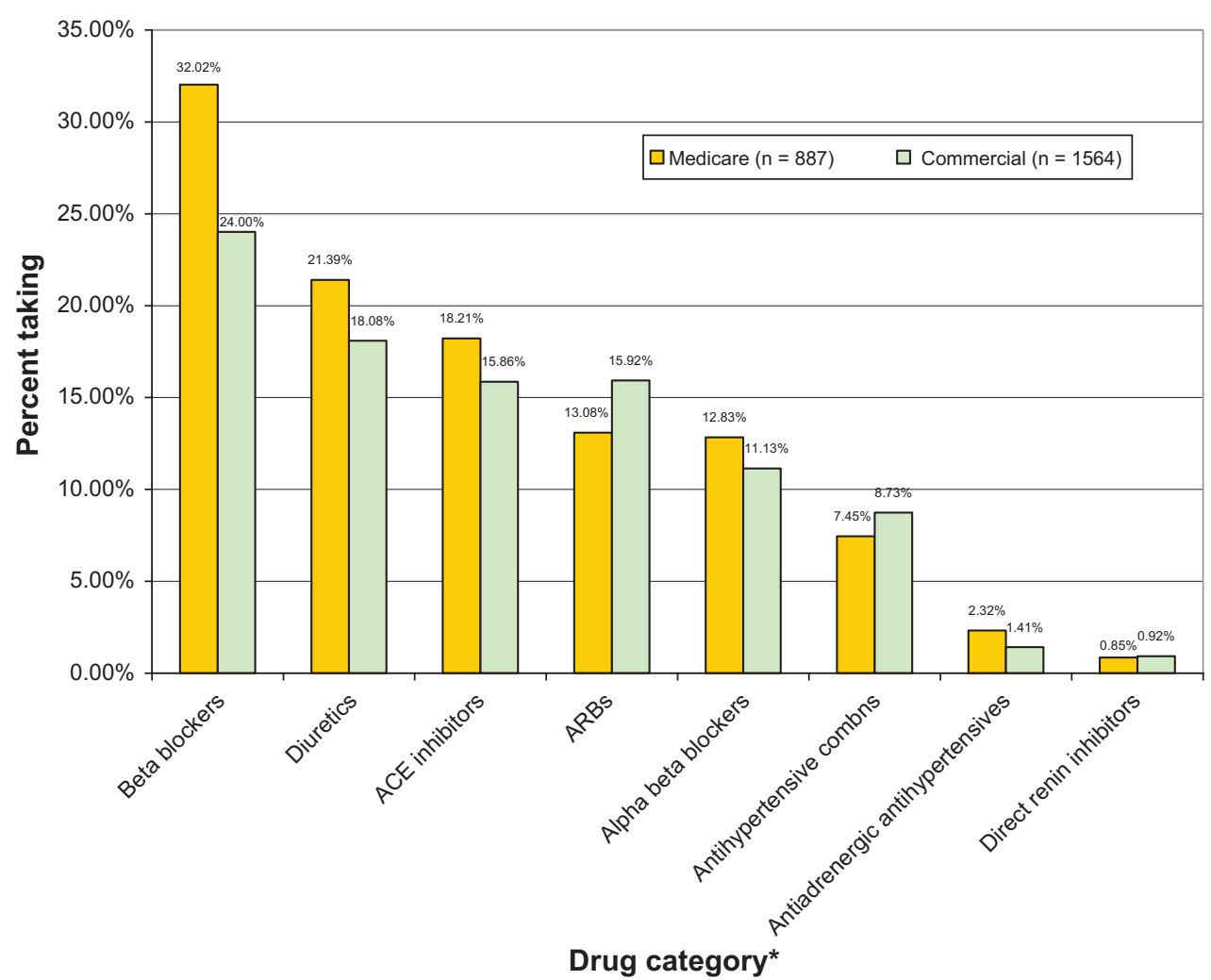

Figure I Distribution of respondents in the antihypertensive drug classes for Medicare-insured and commercially insured respondents. Notes: *Represents drug class of target drug. Diuretics drug class excludes those taking only furosemide for hypertension.

respondents were "too busy sometimes" $(P=0.0002)$, $6.6 \%$ of commercially-insured and $4.1 \%$ of Medicareinsured respondents were "traveling and forgot to pack their medication" ( $P=0.0939$ ), and $<2 \%$ of each group reported they were not able to perform their daily routine $(P=0.3628)$.
Copays were a barrier for $<5 \%$ of each group $(P=0.0273$, Table 4). Medicare members were 7.60 times more likely to report not being able to make it to the pharmacy as compared with commercial enrollees. Medicare members were also 1.62 times more likely to report "other reasons" as a barrier as compared with commercial respondents. Because "other

Table 3 Barriers to adherence for Medicare-insured and commercially-insured survey respondents

\begin{tabular}{|c|c|c|c|c|c|c|c|c|}
\hline & \multicolumn{2}{|c|}{$\begin{array}{l}\text { Medicare-insured } \\
\text { respondents } \\
(n=490) \\
\end{array}$} & \multicolumn{2}{|c|}{$\begin{array}{l}\text { Commercially- } \\
\text { insured respondents } \\
(n=907)\end{array}$} & \multirow[t]{2}{*}{$\begin{array}{l}\text { Adjusted } \\
\text { OR }\end{array}$} & \multirow[t]{2}{*}{$95 \% \mathrm{Cl}$} & & \multirow[t]{2}{*}{$P$ value } \\
\hline & $\mathbf{n}$ & $\%$ & $\mathbf{n}$ & $\%$ & & & & \\
\hline Copay was too high & 25 & 5.1 & 32 & 3.53 & 2.407 & 1.103 & 5.249 & 0.1582 \\
\hline Drug had too many side effects & 3 & 0.61 & 8 & 0.88 & 0.473 & 0.076 & 2.622 & 0.5886 \\
\hline Drug was not effective & 0 & 0 & I & 0.11 & NA & & & 0.9505 \\
\hline I just forgot & 318 & 64.9 & 665 & 73.32 & 0.69 & 0.491 & 0.853 & 0.001 \\
\hline $\begin{array}{l}\text { Blood pressure is under control } \\
\text { without medication }\end{array}$ & 12 & 2.45 & 10 & 1.1 & 1.457 & 0.432 & 5.25 & 0.0602 \\
\hline $\begin{array}{l}\text { Blood pressure is under control } \\
\text { with other things }\end{array}$ & 0 & 0 & 4 & 0.44 & NA & NA & & NA \\
\hline Too busy & 13 & 2.65 & 168 & 18.52 & 0.168 & 0.087 & 0.326 & $<0.000$ I \\
\hline Could not make it to pharmacy & 31 & 6.33 & 8 & 0.88 & 7.59 & 3.461 & 16.645 & $<0.000$ I \\
\hline Other reason & 113 & 23.06 & 79 & 8.71 & 1.618 & 1.032 & 2.535 & $<0.0358$ \\
\hline
\end{tabular}

Notes: *Reference group comprises commercial respondents and is adjusted for age and gender. Survey question: "Can you tell me a little bit about why you missed your dose of [target drug] that time?" (Note that only $60 \%$ of nonadherent patients reported missing a dose, suggesting that $40 \%$ were unaware of their nonadherence). Sample size for each question represents those members who responded to the specific question they were directed to, and therefore can vary from question to question. Abbreviations: NA, sample size too small to conduct adjusted analysis; OR, odds ratio; Cl, confidence interval. 
Table 4 Self-reported aids to overcoming adherence for Medicare-insured and commercially-insured survey respondents

\begin{tabular}{|c|c|c|c|c|c|c|c|c|}
\hline & \multicolumn{2}{|c|}{$\begin{array}{l}\text { Medicare-insured } \\
\text { respondents } \\
(\mathrm{n}=30 \mathrm{I}) \\
\end{array}$} & \multicolumn{2}{|c|}{$\begin{array}{l}\text { Commercially- } \\
\text { insured respondents } \\
(n=490)\end{array}$} & \multirow[t]{2}{*}{$\begin{array}{l}\text { Adjusted } \\
\text { OR }\end{array}$} & \multirow[t]{2}{*}{$95 \% \mathrm{Cl}$} & \multirow[t]{2}{*}{$P$ value } & \\
\hline & $\mathbf{n}$ & $\%$ & n & $\%$ & & & & \\
\hline Has support of family or friends & 27 & 8.97 & 22 & 4.49 & 1.293 & 0.584 & 2.86 & 0.526 \\
\hline Sets a reminder & 5 & 1.66 & 14 & 2.86 & 0.595 & 0.162 & 2.189 & 0.5351 \\
\hline Part of a daily routine & 140 & 46.51 & 256 & 52.24 & 1.084 & 0.739 & 1.589 & 0.6807 \\
\hline Just good about remembering & 68 & 22.59 & 121 & 24.69 & 0.624 & 0.397 & 0.98 & 0.0408 \\
\hline Other & 61 & 20.27 & 77 & |5.7| & 1.474 & 0.894 & 2.431 & 0.1281 \\
\hline
\end{tabular}

Notes: *Reference group comprises commercial respondents and is adjusted for age and gender. Survey question: "What helps you to remember to take your medication?" Sample size for each question represents those members who responded to the specific question they were directed to, and therefore can vary from question to question.

Abbreviations: $\mathrm{OR}$, odds ratio; $\mathrm{Cl}$, confidence interval.

reasons" represented between $8 \%$ and $23 \%$ of all responses in each group, a qualitative examination of the other category (Figure 2) revealed that the predominant reason for missing a medication dose was disruption in daily routine, including a change of routine on weekends or on vacation, or unintended behaviors, such as falling asleep or being late for work.

\section{Self-reported aids to overcoming nonadherence}

Approximately $50 \%$ of respondents in each group reported "part of their daily routine" as helping them remember to take their target medication $(P=0.1181)$. About $25 \%$ of members in each group reported they were "good about remembering" to take their medication $(P=0.5009)$. Medicare members were 2.02 times more likely to report having family or friends' support to help remind them about taking their target medication as compared with commercially-insured respondents. Over 70\% cited other reasons, such as using a pill box, while $14 \%$ reported keeping medications in a visible place as a reminder aid.

\section{Means of medication purchase}

A majority of respondents in each group (73.5\% commercial and $57.7 \%$ Medicare) purchased their target medication through the pharmacy benefit of their health plan. Between $11.6 \%$ and $15.5 \%$ said they receive their target drug directly

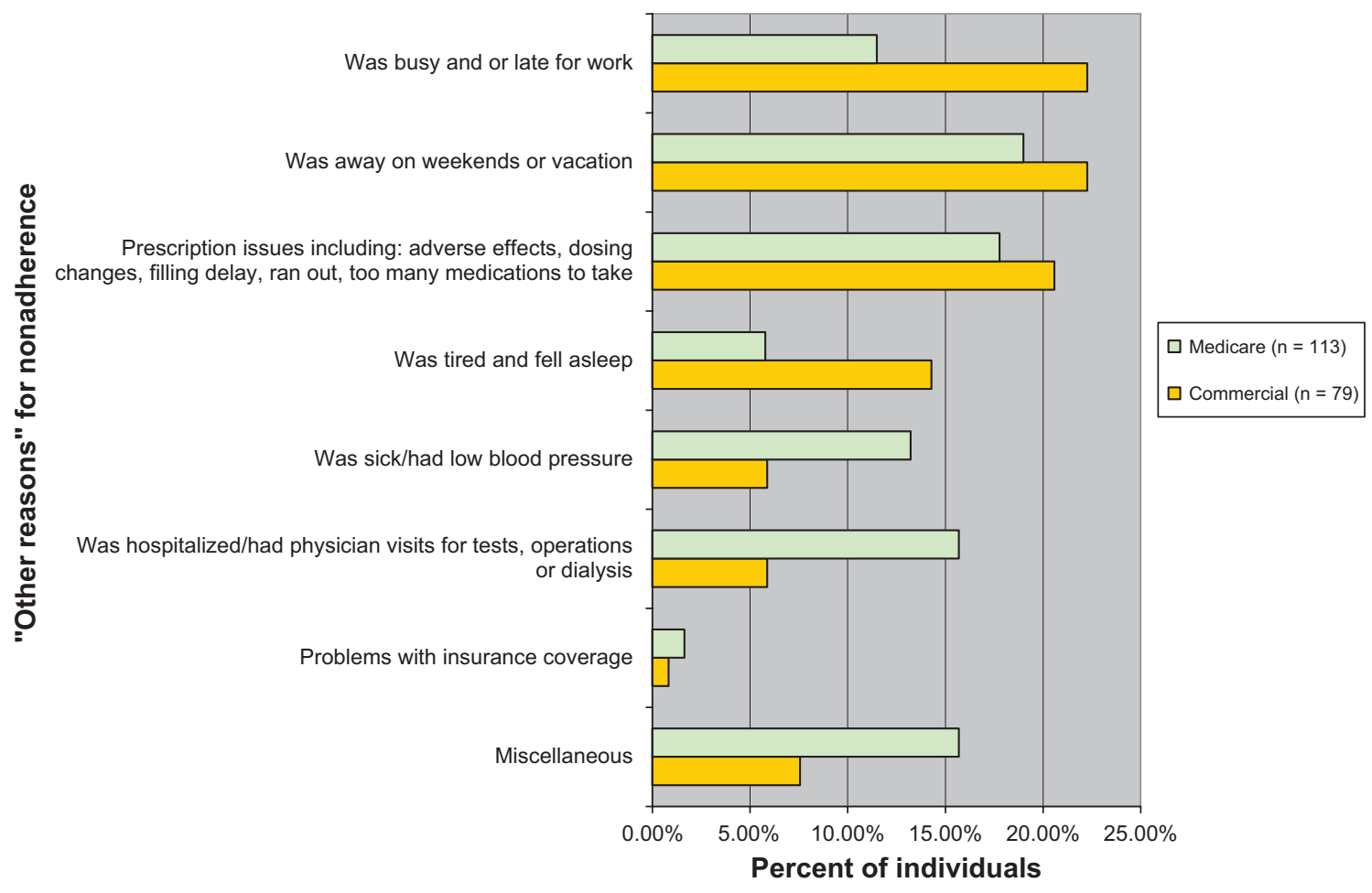

Figure 2 Self-reported "other reasons" for nonadherence by Medicare-insured and commercially insured respondents. Survey question: Can you tell me a little bit about why you missed your dose of the target drug that time? Responses to "other reasons". 
as samples from their physician (and therefore may have been considered nonadherent).

\section{Use of mail-order pharmacy services}

For respondents who reported they were late in picking up their prescription, $35.1 \%$ of Medicare-insured and $48.1 \%$ of commercially-insured respondents $(P<0.0001)$ reported that they would be interested in using mail-order pharmacy services to obtain their target medication.

\section{Preferred method of receiving educational materials}

A majority of respondents in both groups (62.4\% commercial and $75.1 \%$ Medicare) preferred to receive educational material through regular mail. A higher percentage of commercially-insured respondents $(15.1 \%)$ as compared with Medicare-insured respondents $(3.8 \%)$ preferred to receive information via email. Approximately $12 \%$ of each group preferred to receive information through calls from a live agent, while $<2 \%$ wanted to receive calls through an automated response voice system.

\section{Discussion}

These data highlight several important issues. First, despite selecting a sample of hypertensive patients who had been determined to be nonadherent to at least one of their antihypertensive medications, our results indicate that only about $60 \%$ of respondents reported missing a dose of their medication, and an additional 15\% reported picking up their pills a few days late. Thus, only $75 \%$ of the respondents reported nonadherence to the target drug. This is in line with the rate of underreporting cited in a study assessing the presence of discrepancies by comparing medication bottles with medication records, ${ }^{17}$ in which discrepancies between recorded and reported medication use were observed for $76 \%$ of the study population.

Second, differences in age did not appear to influence nonadherence, as suggested by the similar adherence rates for commercially-insured and Medicare-insured patients despite the significant age difference. Previous research has also shown that age does not predict adherence reliably. ${ }^{18}$

The most important finding is that unintentional nonadherence appears to be the primary form of nonadherence for the hypertensive patients in our study. A majority of respondents reported simply forgetting to take their medications if they were too busy or if they experienced disruptions in their daily routine, such as being late for work, traveling, vacationing, being sick or hospitalized, or undergoing medical tests that required them to discontinue their medications. More Medicare members reported accessrelated issues of not being able to make it to the pharmacy compared with commercial members, while more commercial members reported missing a dose of their medication because they were too busy.

Previous adherence studies in hypertensive populations were based on the notion that medication-taking behavior depends on the value a patient places on a particular outcome (eg, the effects of uncontrolled hypertension) and the patient's estimate of the likelihood that a particular activity (use of antihypertensive medications) will produce a desired outcome (controlling hypertension). ${ }^{19}$ We did not measure the value our study subjects placed on particular outcomes, nor did we assess their estimates of the likelihood that the use of antihypertensive medications would produce a desired outcome. Therefore, we cannot report any findings that confirm or refute the influence of patient values and expectations on medication adherence. Rather, our findings indicate that medication adherence appears to be a patterned behavior established through the creation of a routine and a reminder system for taking the medication. Disruption of this routine, due to external events and triggers, leads to nonadherence.

As a first step in designing meaningful interventions, health care providers can assess daily lifestyle and demands for hypertensive patients to determine the likelihood of disruption by asking simple questions such as, "Can you take your medications on a regular basis"? or "What is your routine in taking your medications"? The information from this assessment can help providers determine which dosing schedule (and thereby which medication) a patient is capable of being adherent to, along with potential times and locations to develop a regular pattern of taking medications.

Information about a patient's lifestyle can be used to develop strategies to minimize unintentional nonadherence. Such strategies could include telephone or email reminders, or the use of medication charts that patients can refer to and check off each time they take their medication. In a review of interventions designed to improve adherence for cardiovascular medications, five randomized clinical trials which utilized prompting mechanisms such as daily and weekly telephone calls, video telephone calls, mailed reminders, and electronic medication reminder caps were examined. ${ }^{19}$ In three of these trials, prompting mechanisms significantly increased adherence compared with usual care.

Providers can also reinforce the importance of taking antihypertensive medications to patients so that their beliefs 
about the necessity of these medications continue to be maintained and allow patients to make fewer unintentional decisions about taking their medications.

Our study had several limitations. First, we were not able to examine the impact of nonadherence on actual blood pressure readings. We did not have access to patient charts and so were unable to determine whether respondents who were nonadherent to their target medication were also unable to control their hypertension. As a proxy for this measure, one of the follow-up questions asked was, "Do you know if your blood pressure is under control"? Over $96 \%$ of all respondents responded affirmatively. However, without verifying this information with clinical data, we were not able to assess the clinical impact of nonadherence in our study sample.

Second, we decided to use only the antihypertensive medication associated with the lowest adherence rate in the telephone survey, irrespective of how many other antihypertensive medications respondents were taking. The choice of the primary hypertensive drug was based on the most recent hypertensive drug prescribed to a member in order to minimize recall bias among the respondents. Given the possibility that members may have switched between hypertensive drugs, which happens frequently for clinical reasons, we chose to focus on one recent and primary hypertensive drug to ensure that respondents would be able to recall as much detail as possible. Pharmacy claims revealed that, on average, respondents were taking 2.6 hypertensive medications, with a mean adherence rate of $85 \%$. The overall adherence to other antihypertensive medications excluding the target drug was high for our study sample. Selecting a sample whose adherence rates were $<80 \%$ for all antihypertensive drugs may have been a better approach for identifying nonadherent hypertensive patients and could have yielded different results. Third, we did not survey hypertensive patients with high adherence $(\geq 80 \%)$ in order to compare their responses with those of patients with low or moderate adherence. This comparison would have provided a more complete picture of the barriers that affect nonadherence and should be undertaken in future studies.

Fourth, we present results of analyses that adjusted only for age and gender when comparing the group differences with regard to barriers to adherence and self-reported aids to overcoming nonadherence. We did conduct an adjusted analysis that controlled for a number of additional variables including age, gender, RxHCC risk score, adherence rate for target hypertensive medication, total number of antihypertensive medications, total number of all prescriptions, and member out-of-pocket expenditures for all medications, which were the variables available from claims data we felt could most likely explain some of the betweengroup differences. More information about occupation, income, and education for the two groups of patients (under and over 65 years of age) would have allowed for more meaningful interpretation of the data.

Fifth, we used several skip patterns and follow-up nested questions in our telephone survey in order to understand the reasons why patients reported a specific barrier. The more involved study design resulted in small sample sizes for some of the follow-up questions, thus limiting our ability to conduct more detailed and multivariate analyses comparing the commercially-insured and Medicare-insured groups.

Sixth, the inclusion of an insured study population may not be representative of other patient groups. And finally, the lack of sampling of a comparison group with high MPR for the same target drugs diminishes the utility in identifying factors associated with nonadherence.

\section{Conclusion}

Our study findings have furthered our understanding of what drives nonadherence in hypertensive patients. Patients nonadherent to at least one hypertensive medication appeared to exhibit unintentional nonadherence. The primary reason for unintentional nonadherence was forgetfulness. Factors that triggered forgetfulness appeared to be a disruption in daily routine, such as a change in routine on weekends or on vacation, or other behaviors, such as falling asleep or being late for work. Other reasons included missing a medication dose due to sickness, hospitalization, or a medical procedure that required them to stop taking their medication. These findings can be used to design interventions that take into account patients' daily lifestyles and to design medication treatment plans that cause the least disruption to the patient.

\section{Disclosure}

This study was funded by Novartis Pharmaceuticals Corporation. DAB and JJD are employees of Novartis Pharmaceuticals Corporation.

\section{References}

1. Mapes RE. Physicians drug innovation and relinquishment. Soc Sci Med. 1977;11:619-624.

2. Sackett DL, Haynes RB, Gibson ES, et al. Randomized clinical trial of strategies for improving medication compliance in primary hypertension. Lancet. 1975;1:1205-1207.

3. Sabate E. Adherence to long term therapies: Evidence for action. Geneva, Switzerland: World Health Organization; 2003. 
4. Andrade SE, Kahler KH, Frech F, et al. Methods for evaluation of medication adherence and persistence using automated databases. Pharmacoepidemiol Drug Saf. 2006;15:565-574.

5. Sikka R, Xia F, Aubert RE. Estimating medication persistency using administrative claims data. Am J Manag Care. 2005;11:449-457.

6. DiMatteo M, Giordani P, Lepper H, et al. Patient adherence and medical treatment outcomes. Med Care. 2002;40:794-811.

7. Mazzaglia G, Ambrosioni E, Alacqua M, et al. Adherence to antihypertensive medications and cardiovascular morbidity among newly diagnosed hypertensive patients. Circulation. 2009;120:1598-1605.

8. Cameron C. Patient compliance: Recognition of factors involved and suggestions for promoting compliance with therapeutic regimens. J Adv Nurs. 2007;24;244-250.

9. Kingsnorth D, Wilkinson S. Patient compliance with medication regimens after discharge from palliative care. Int J Palliat Nurs. 1996;2: 144-148.

10. Lehane E. An examination of intentional and unintentional aspects of medication non adherence in patients diagnosed with hypertension. J Clin Nurs. 2007;16:698-706.

11. Harmon G, Lefante J, Wood-Krousel M. Overcoming barriers: Role of providers in improving patient adherence to antihypertensive medications. Curr Opin Cardiol. 2006;21:310-315.
12. Staffan S, Kjellgren KI, Ahlner J, Saljo R. Reasons for adherence with antihypertensive medications. Int J Cardiol. 2000;76:157-163.

13. Turner BJ, Hollenbeak C, Weiner MG, et al. Barriers to adherence and hypertension control in a racially diverse representative sample of elderly primary care patients. Pharmacoepidemiol Drug Saf. 2009;18: 672-681.

14. Dragomir A, Côté R, Roy L, et al. Impact of adherence to antihypertensive agents on clinical outcomes and hospitalization costs. Med Care. 2010;48:418-425.

15. Care Management International: Homepage. Available at: http://www. caremanagementinternational.com/. Accessed November 14, 2010.

16. Robust J, Levy JM, Ingber MJ. Diagnosis based risk adjustment for Medicare prescription drug plan payments. Health Care Financ Rev. 2007;28:15-30.

17. Bedell SE, Jabbour S, Goldberg S. Discrepancies in the use of medications. Their extent and predictors in outpatient practice. Arch Intern Med. 2000;160:2129-2134.

18. Jacob-Dunbar J, Stephens-Mortimer MK. Treatment adherence in chronic disease. J Clin Epidemiol. 2001;54:S57-S60.

19. Johnson MJ, Williams M, Marshall ES. Adherent and non adherent medication taking behavior in elderly hypertensive patients. Clin Nurs Res. 1999;83:318-335. 


\section{Appendix}

Confirmation of presence of hypertension and use of target drug: Sampling of survey questions and responses:

1. I see that recently you may have been prescribed [target drug], which is commonly used to lower blood pressure. Can you tell me if you are currently taking [target drug] for high blood pressure?

Individuals were asked to respond by choosing one of these options: A Yes, still taking [target drug] for high blood pressure, B Yes, taking [target drug] but do not have high blood pressure or do not know if [target drug] is for high blood pressure, C No, not taking [target drug] but taking another drug for high blood pressure, or D No, not taking [target drug] anymore.

\section{Dosing pattern}

For those respondents who selected responses $1 \mathbf{A}, \mathbf{1 B}$, or 1C, the following items were used to assess dosing pattern of the target drug:

2. How many times per day do you take this medication? Individuals were asked to respond by choosing one of these options: A once, $\mathbf{B}$ twice, $\mathbf{C}$ three times, $\mathbf{D}$ other.

3. At what time of the day do you take the medication? Individuals were asked to respond by choosing one or more of these options: A morning, B afternoon, C evening.

\section{Self-reported nonadherence}

For those respondents who answered Question 2 and Question 3, the following items were used to determine self-reported nonadherence to the target medication:

4. Do you ever miss taking a dose of your [target drug] here or there?

Individuals were asked to respond by choosing one of the following options: A yes or $\mathbf{B}$ no.

Those who answered "no" to Question 4 were asked:

5. "Have you ever picked up your prescription a couple of days after your pills ran out?

Individuals were asked to respond by choosing one of the following options: A yes or B no.

\section{Barriers to adherence}

The following items were used to determine barriers to selfreported nonadherence to the target medication for members who answered "yes" to Question 4:*

6. Can you tell me a little bit about why you missed your dose [of the target drug] that time?
Individuals were asked to respond freely (ie, in an open-ended format), after which the interviewer summarized the respondent's explanation and asked him or her to confirm that the dose was missed for up to three of the following reasons: A copay was too high, B drug had too many side effects, $\mathbf{C}$ drug did not work or there was no effect from taking the drug, D I just forgot, E I didn’t need the medication because my high blood pressure is under control without help, F I didn't need the medication because I control my high blood pressure with other things (eg, exercise, diet), G I was too busy (other things more important), H I could not make it to the pharmacy, or I other reason.

*Follow-up questions to each of the specific barriers cited were also conducted but are not presented here.

Individuals who reported that they "just forgot" to take their target medication (6D) were asked:

7. Can you tell me some of the reasons why you may forget to take your medication?

Individuals were asked to respond by choosing up to three of the following options: A I am too busy sometimes, B I was traveling and forgot to pack the medication, C I was not able to perform my daily routine, D It just slipped my mind, E Random event happened and I could not take my medicine, or $\mathbf{F}$ other reason.

\section{Means of medication purchase}

For those who answered "no" to Question 5, the following item was used to determine where respondents purchased/ obtained their target medication:

8. How do you most typically obtain your prescription for [target drug]?

Please answer yes or no to the following: A Do you ever receive drug samples from your doctor?, B Does your pharmacy insurance plan cover your prescriptions?, C Do you sometimes use another type of pharmacy insurance?, D Do you use a discount program offered by drug manufacturers?, E Do you ever buy your prescription through a special store offer, like the four dollar generic program at Walmart or Target stores?, or $\mathbf{F}$ None of the above.

\section{Self-reported aids to overcoming nonadherence}

For members who responded to Question 7, the survey item below was used to determine self-reported aids to overcoming nonadherence to the target medication:

9. What helps you remember to take your medications? 
Individuals were asked to respond by choosing one of the following options: A I have family or friends' support, B I set a reminder, C It's part of a daily routine, D I'm just good about remembering, or $\mathbf{E}$ other.

\section{Use of mail-order pharmacy services}

For those members who answered "yes" to Question 5, the following item was used to determine whether they would be interested in using mail-order pharmacies to obtain their target drug:

10. If you had an option to use the mail-order pharmacy, would you be interested?

Individuals were asked to respond by answering $\mathbf{A}$ yes or $\mathbf{B}$ no.

\section{Preferred method of receiving educational materials}

At the end of the call, the following item was used to determine respondents' preferred method of receiving educational materials about blood pressure:

11. Finally, if your insurance plan were to provide you with educational material, which delivery method would you prefer?

Individuals were asked to respond by choosing one or more of the following options: A Via regular mail (newsletters and letters), $\mathbf{B}$ via email, $\mathbf{C}$ via phone call with a live person, $\mathbf{D}$ via phone call through an automated voice system, or $\mathbf{E}$ none of the above - please don't send me any information.

\section{Publish your work in this journal}

Patient Preference and Adherence is an international, peer-reviewed, open access journal focusing on the growing importance of patient preference and adherence throughout the therapeutic continuum. Patient satisfaction, acceptability, quality of life, compliance, persistence and their role in developing new therapeutic modalities and compounds to optimize clinical outcomes for existing disease states are major areas of interest. This journal has been accepted for indexing on PubMed Central. The manuscript management system is completely online and includes a very quick and fair peer-review system. Visit http://www.dovepress.com/ testimonials.php to read real quotes from published authors. 